\title{
Web-Based Portal for Sharing Information through CAD/PLM Software during the Eco-product Development Process
}

\author{
Idai Mendy Mombeshora and Elies Dekoninck
}

\begin{abstract}
Against the backdrop of increasing global demand for environmentally sustain-able products, the integrated software platform for Green ENgineering dESIgn and product sustainability (G.EN.ESI) project aims to develop a software platform, for use in conjunction with CAD/PLM software, which simplifies the process of integrating environmental and economic requirements into the product development process. A key component of the platform, and the main focus of this paper, is its unique web based supply chain portal that has the ability to obtain information directly from the supply chain. This paper details the work undertaken in the early stages of its development to explore possible portal architectures based on their strategic alignment with goals of firms using it. Based on research and analysis of past and existing supplier portals and data collected through an online survey and a case study, four possible architectures of the web portal were derived using scenario planning and their viability was tested using use cases and wind-tunnelling. The results suggest that although all generated scenarios are viable, the one in which multiple buyers and multiple suppliers interact with a single web portal is the most favourable. The consolidation in buyers and suppliers mitigates any bargain power related issues that might arise, while making way for the possible development of an industry wide information sharing standard for eco-design. Moving forward, the project aims to gain a better understanding of supplier collaboration in new product development through the use of the portal by exploring the nature of the information being shared, the roles that users of the portal play and any competitive conditions associated with the use of the portal.
\end{abstract}

\section{Introduction}

With organisations experiencing increased social and regulatory demands to behave in an environmentally conscious manner, environmental impact is fast becoming a factor considered on par with cost, functionality and value during the product development process. Against this back drop, some organisations are enhancing their competitiveness by improving their environmental performance through the mitigation of the environmental impact of their production and service activities (Bacallan, 2000). However, others view these new requirements as mandates or burdens that slow development while ramping up cost, detracting from the main business of the company. As a result, environmental aspects are often considered an afterthought, resulting in delays and added costs as changes are made after the late addition of environmental 
requirements into the development process (Handfield et al., 2001). With its roots in concurrent engineering; three dimensional concurrent engineering (3DCE) holds great promise for integrating environmental considerations into the product development process. 3DCE is the notion that the simultaneous design of product, process and supply chain, through links between internal functions and participation with external partners, leads to improved operating performance (Fine, 1998). Although relatively new in practice, as companies are begin to embrace it, 3DCE appears to be a lens for demonstrating that eco-design efforts can support both traditional and environmental product development goals (Ellram et al., 2008).

As the environmental performance of a product is the amalgamation of its environmental impact through all the stages of its lifecycle, from the extraction of raw materials to its end of life, it is dependent on the totality of the supply chain in both upstream and downstream directions throughout its lifecycle. During the product development process, it is necessary to have as much information as possible pertaining to the environmental performance of the various supply chain partners and the products and services they provide. With product lifecycle management (PLM) systems gaining acceptance as means for managing information about a product throughout its lifecycle (Sudarsan et al., 2005), they hold the promise of seamlessly integrating and making available all of the information produced throughout all phases of a product's life cycle to everyone in an organisation and through the product network.

Set within the context of the household appliance industry and through the adoption of the 3DCE and PLM approaches, the integrated software platform for Green ENgineering dESIgn and product sustainability (G.EN.ESI) project is a European Union Seventh Framework project that aims to achieve a $30 \%$ reduction in lifecycle energy use and a 50\% reduction in industrial waste in household appliances produced based on the choices made through its software platform and eco-design methodology. For the successful integration of the G.EN.ESI platform into CAD/PLM software, it is paramount to ensure that there is an accurate and reliable flow of information from various supply chain partners, including suppliers, product dismantlers and distributors, to the software. This vital flow of information from the supply chain into the design process via the G.EN.ESI platform will be realised through a unique webbased supply chain portal. Since internet communication technologies gained popularity as a means of simplifying business to business communications, supplier portals have been found to promote information sharing and coordination of operational flows (McIvor and McHugh, 2000), sup-port supplier management and create a sense of community among buyers and suppliers; while increasing the stability of relationships and suppliers' loyalty to their customers (Roberts, 1999). It is this collaborative potential within web portals that the G.EN.ESI project is looking to harness.

In this paper, the G.EN.ESI project will be presented, with particular focus on the initial steps in the development of the web portal which can be viewed as the evolution of the supply chain web portal from its traditional role as an e-procurement tool into an information sharing tool. The web portal's development will be presented after a brief discussion of information sharing and inter-organisational systems. 


\subsection{Importance of Inter-organisational Information Sharing during the Product Development Process}

Information sharing can be applied to almost all core domains of corporate operational activities. Ranging from customer chains where information can aid in the formulation of customer experience strategies, to exchanges within the development chain where information is shared within product design and product lifecycle management activities and supply chain information exchanges that lead to greater visibility and responsiveness (Yu et al., 2001). Typically, information sharing within the supply chain is associated with maximising responsiveness and efficiency while minimising cost, with the relationships formed handled by the procurement and/or logistics department; while, information sharing within the product development chain is allied with the acquisition of resources and capabilities to improve product offerings, with the collaborative relationships formed more likely to have a research and development focus.

On the one hand, there is Kanter's notion of collaboration advantage, defined as " $a$ significant leg up in a global economy due to a firm's well developed ability to create and sustain fruitful collaborations" (Kanter, 1994), which is associated with the product development chain; while on the other, there is the resource-based theory view that one source of differential performance between firms is the way in which they organise exchange activity (Conner and Prahalad, 1996), which is related to the supply chain. Therefore, it would seem logical to then deduce that amalgamation of the two forms of information sharing would result in advantages gained through the unified use of the formed relationships, enriching the depth and quality of information shared via both design and supply chains. With particular focus on design chains and collaborative design, utilising supply chain information sharing relationships and methods within the product development process would offer a means of augmenting the match between product and process, which most companies accomplish through concurrent engineering, with an additional consideration of supply chain configuration.

\section{The G.EN.ESI Project}

Through the development of a software design tool (G.EN.ESI platform) and supporting eco-design methodology (G.EN.ESI methodology), the G.EN.ESI project aims to address the lack of easy to use and robust tools for environmental evaluation at the engineering design stage. Currently available tools are either too qualitative/subjective to be used by designers with limited experience, or too quantitative, costly and time consuming for use during the early stages of the product development process (Boks, 2006). Moreover; these tools are usually stand alone and do not allow for easy integration with traditional design tools. The shortcomings of current ecodesign procedures and tools mean they fail to offer practical solutions for day-to-day 
use as they only achieve limited industry penetration (Lofthouse, 2006). The main objective of the project is to supply a plat-form that can be completely integrated with other main design tools, such as CAD and PLM software, which helps designers make ecological design choices without losing sight of cost and typical practicalities of industry.

The G.EN.ESI platform architecture will be based on the integration of various tools into the same structure, with the tools communicating to support the entire product design process; an example of a tool is the S-LCC analyst tool which will evaluate the whole product lifecycle. Each tool within the platform will examine design choices from a specific point of view while simultaneously possessing the ability to provide information to the designer on environmental issues. This connection between the tools will allow for an immediate check of the congruence of the choices with other key design parameters. Additionally, environmental decisions made by the designer will be supported by a case-based reasoning tool which will utilise knowledge stored from previously successful cases to suggest possible environmental improvements.

\subsection{How the Supply Chain Portal Supports the G.EN.ESI Platform}

A reliable input of accurate data and information is central to the success of the software platform; the platform will manage data through relational databases structured such that they align with the most common databases which support software tools used by the companies. The G.EN.ESI databases will not only inherit data from local company software tools (CAD/PLM) but will also collate data from various members of the supply chain through the use of a web-based portal. Using information that the supply base inputs into the portal, the platform tools assesses the environmental impacts and cost of various options, such as materials, components, processes etc., allowing the designer to select the most convenient in terms of environmental aspects. The use of the web portal will encourage sustainability competitiveness within industry, while stimulating eco-efficiency throughout the whole supply network.

Figure 1 shows the structure of the web portal proposed by the project and how the portal interacts with the software platform. An example of how the portal and platform interact when used by a designer is as follows: a member of the supply chain uploads information into the portal regarding a component, including weight of component, geographical location of the production plant and transport used to ship it. When the designer selects this component during the design process, the $0 \mathrm{~km}$ tool within the platform automatically downloads all the information regarding the transport scenario from the portal. The $0 \mathrm{~km}$ tool collates transport information relating to all the chosen components within the design, it is this information that is used as part of the environmental impact (S-LCA) and cost (S-LCC) calculation along with calculations made from other tools such as DFEE and LeanDFD. The results of these calculations are then presented to the designer. 


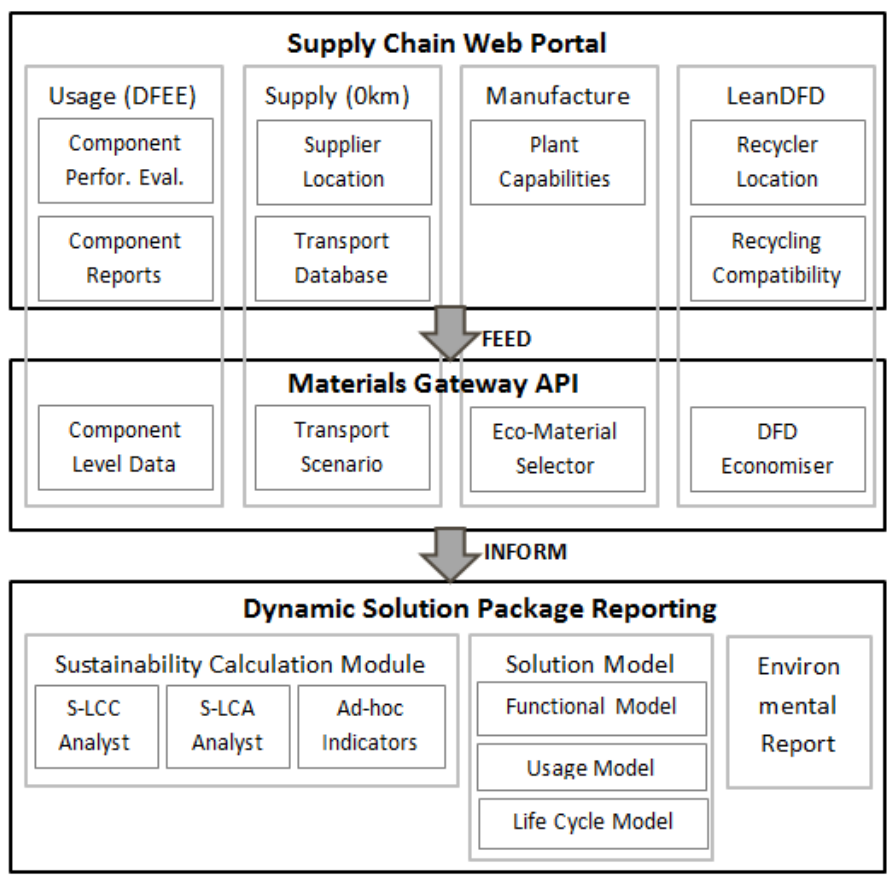

Fig. 1. Structure of the G.EN.ESI platform's web portal

\section{Supply Chain Portal Development Objectives}

To ensure effective deployment of the supply chain portal, it was essential at this early stage in its development to explore possible portal architectures based on the impact that the nature of shared information, who it is shared with, how it is shared and when it will be shared would have. Based on this, the following specific aims were created for this paper:

1. Explore possible portal architectures and resultant strategic scenarios for the web portal - to ensure that the G.EN.ESI web portal's functional requirements align with the strategic implications it would have on users.

2. Develop possible use cases for the web portal and use them to test the viability of the architectures and strategic scenario - to gain an understating of how the portals would perform in various situations.

3. Classify existing compliance web portals based on the developed scenarios and architecture -a way of understanding what is already available and how it relates to the proposed G.EN.ESI platform

\section{$4 \quad$ Research Methodology and Results}

To ensure that the architectures, scenarios and use cases developed are truly representative of what is likely to transpire in industry, it was necessary that their development was based 
on information gathered from potential platform users. Due to the dual nature of the collaboration requirements and to ensure that both options are sufficiently researched, a twophase data collection process was formulated and executed. Firstly, data was collected through an online administered survey aimed at understanding the nature of supplier collaboration in new product development (SCNPD) with particular focus on the perspective of suppliers. The survey garnered 76 responses from individuals involved in SCNPD projects across a number of engineering industries. The second phase, whose aim was to acquire a better understanding of the acceptability of the portal's proposed role, the climate under with it would be used and the profile of a typical platform user, involved an indepth case study of a G.EN.ESI project industrial partner. Table 1 summarises some key results from the two phases of data collection.

Table 1. Key results from data collection phase

\begin{tabular}{|l|}
\hline Online Survey \\
\hline - Most cited factors/qualities to consider when selecting a partner were trust and \\
reliability, openness and mutual support, congruence of goals and relationship. \\
- When asked which was more important, trust or contracts, $50 \%$ of the respon- \\
dents said trust and the other $50 \%$ said they were both equally important. \\
- Most frequently faced challenges when collaborating were relationship man- \\
agement, aligning goals and objectives and financial burden. \\
- Most difficult challenges faced when collaborating were dealing with failed \\
relationships, relationship management and aligning goals and objective \\
- Effects of failure were relationship breakdown and end of collaborations. \\
- Most projects only exchange information and knowledge that is essential. \\
\hline Case Study \\
- Biggest concern with portal is the security of the information that is shared. "I \\
don't know if I can trust sharing information over the internet, how can you be \\
sure that only the people you want to can see the information you upload?" \\
- Cited that the fear that information would get abused, used for anything that has \\
not been agreed upon, is a concern. "It is not like if you are not happy with what \\
they are doing with the information you can take it back". \\
- "Getting information from our suppliers is hard enough as it is, even informa- \\
tion that they possess; they usually refer us to a data sheet on the website." \\
- "If the people we supply to asked us for the information that we would likely \\
have to ask for from our suppliers, we would not be able to provide it."
\end{tabular}

\section{Developing Portal Architectures and Strategic Scenarios}

To determine various strategic scenarios relating to the supply chain web portal, the technique of scenario planning was used. Scenario planning is a futures technique that is used to generate different scenarios that represent possible futures associated with different trends and events to help develop policies and strategies that are robust and flexible (Schoemaker, 1995). In this case, scenario planning was undertaken to determine the following: 1) the best way to structure the supplier portal, 2) strategic 
implications its structure would have on buyers (firms using the G.EN.ESI platform) and suppliers (supply chain firms that upload in-formation into the portal) and 3) how the way the portal is used varies depending on the strategy implemented. The key question, central to the scenario planning, was as follows: "what is the best and most viable way of structuring a web based supply chain portal to facilitate information sharing between firms that use the G.NE.ESI platform and members of their supply chain?" Through the analysis of past and present web portals and insights gained from the data collection, drivers and deterrents were generated and used as the basis of the scenario generation. Some of the key driver and deterrents are listed in Table 2.

Table 2. Drivers and deterrents influencing the adoption of web portals

\begin{tabular}{|l|l|}
\hline \multicolumn{2}{|c|}{ Drivers and Deterrents } \\
\hline Impact on financial performance. & Alignment with strategic focus. \\
Impact on other business processes. & Buyer power vs. supplier power. \\
Impact on product development process. & Cost of use. \\
Ease of data input into portal. & Impact on reputation. \\
Availability of required information. & Availability of resources. \\
\hline
\end{tabular}

The two axis method, based on one of the approaches employed by Shell (Foresight, 2009), was then used to generate four contrasting scenarios that are related to the use of web portals by placing a major factor influencing the future of the issue on each of two axes that cross to form four quadrants. It was identified that the major factors influencing the use of the portals were related to the number of companies that would use a single portal; with Multiple Suppliers $\leftrightarrow$ Single Supplier on one axis and Multiple Buyers $\leftrightarrow$ Single Buyer on the other. A four scenarios diagram, with each scenario represented as a series of potential gains and barriers, was then developed; a simplified version is shown in Figure 2.

Following the scenarios development, the main actions that could be taken to manage the risks inherent in each scenario were identified. With so many firms involved with a single portal in Scenario 1, the development of robust ownership rights is a must to ensure that portal is maintained and monitored for misuse. In Scenario 2, as the buyer has access to collated information regarding a number of suppliers, there is scope for misuse, making it is essential to ensure that the buyer does not have sole responsibility of the portal. The portal should also allow suppliers to export information across multiple portals if they have multiple buyers using the G.EN.ESI platform. Scenario 3 requires a function that allows buyers to assign multiple portals to a single software platform and due to the scale of their responsibilities it is paramount to ensure supplier commitment. The main action with Scenario 4 is to ensure that both parties are fully committed and aware of the work involved if they end up associated with multiple portals; additionally, buyers should be able to assign multiple portals to a single platform and suppliers should be able to share information across multiple portals. Regardless of the scenario, it is essential to guarantee the security of information shared through the use of heightened security measures. 
Scenario 1 - Multiple Suppliers/Multiple Buyers

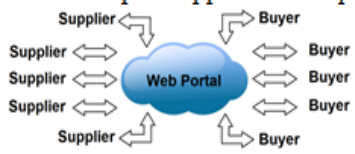

Ease of buying in due to stakeholder count

One portal and multiple users reduces costs

Pooling of resources

Stringent security means required

Ease of use due to information centralisation

Issues of eroded competiveness arise

Formation of an industry wide consortium

Standardisation of eco-information sharing

- Fear of abuse of information

Scenario 3 - Single Supplier/Multiple Buyers

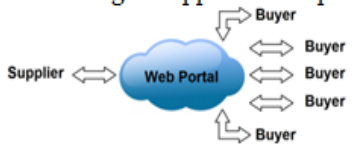

Increased buyer barging power and more incentive for suppliers to be involved

Supplier only inputs data into portal once

Supplier can specify which buyers see what

Stringent security means required

Supplier more comfortable with integrating eprocurement into portal

Strengthened buyer-supplier relationships

Supplier responsible for portal

Increased costs and work load for buyers who interact with more than one portal
Scenario 2 - Multiple Suppliers/Single Buyer

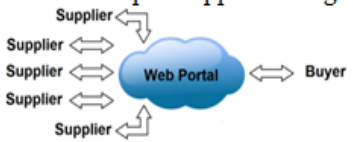

Ease of use due to information centralisation

Buyer builds relationships through portal use

- Reduced costs for buyer due to single portal

Increased costs and work load for suppliers who interact with more than one portal

Fear of abuse of information

Buyer's bargaining power increases as the number of suppliers involved increases

When correctly utilised, portal a strategic differentiator

Scenario 4 - Single Supplier/Single Buyer

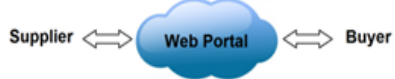

Time consuming information exchange if associated with more than one portal

G.EN.ESI platform interacts with multiple portals

Both parties likely discouraged by tedious nature information exchange

- Less stringent security measures required

Increased implementation and running costs

Integration with e-procurement to increase versatility and usability

Party with the most bargaining power likely to impose their will more successfully

Fig. 2. Simplified four scenarios diagram

\section{Strategic Scenario Viability Based on Portal Use Cases}

Guided by the insights gained during the data collection phase, ten possible use cases were developed. These are possible cases that can arise when the various portal architectures, and their resultant strategic scenarios, are implemented. The list of cases was not exhaustive however; it aimed to encompass a variety of ways that the portal could be used. An example of a use case is as follows: various people from the supplying company have access to the portal. For example, designers of the components being supplied manually enter information into the web portal. There is no one that has been specifically assigned the task of regulating the way in which information is uploaded into the portal.

When set within the context of the strategic scenarios, the use cases can provide useful 'reality checks' on the scenarios, helping to consolidate the overall vision of the web portal with the way it is used and highlight potential risks and challenges. As a result, the use cases were tested against the developed scenarios by mapping them against each other in a matrix (wind-tunnelling) (Foresight, 2009). The focus was on how robust each use case is (can it be performed within the scenario) and the ease of implementation and use. Wind-tunnelling offers a way of determining the viability of 
the scenarios; if all use cases are robust or easy it suggests high viability, only certain use cases are robust or easy suggests medium viability and no robust or easy use cases suggests low versatility.

The results from the wind tunnelling ranked Scenario 1 as highly viable, Scenario 3 as medium-high viability and Scenarios 2 and 4 as medium viability. This suggests that within the context of the G.EN.ESI project, all the scenarios would work, with Scenario 1 being the most favourable as within it all use cases were deemed as at least possible and at most robust and easy. In the kitchen appliance manufacturing industry, which is mainly comprised of SMEs, having one portal as proposed by Scenario 1 offers significant benefits in terms of acquiring supplier buy-in. Through the use of a single portal, multiple buyers and suppliers are consolidated, mitigating any issues that might arise as a result of mismatched bargaining power that is likely to arise between individual buyers and suppliers. As more and more firms within an industry adopt the G.EN.ESI platform, it could provide a consistent method of sharing information resulting in the formation of an industry wide information sharing standard that has a particular focus of eco-design.

\section{$7 \quad$ The Nature of Existing Compliance Supplier Web Portals}

To put the G.EN.ESI portal into context in terms of the evolution of supply web portals, research was undertaken into existing web portals to determine their nature and the scenario which their structure would fall into.

It is undisputable that the use of web portals is on the rise as the popularity of advanced procurement increases; however, due to the difference in the nature of information being exchanged, a shift in focus from traditional procurement portals to compliance portals is required. Examples of such portals include BOMcheck (www.bomcheck.net), in the Scenario 1 category; Freescale (www.freescale.com), in the Scenario 2 category and vendors such as Enovia (www.3ds.com) and Sup-plier Soft (www.suppliersoft.com), who offer self-service portals where suppliers get a single interface to interact with, while the deployed system depends entirely on the user and industry. These portals are generally used to exchange information including environmental compliance, conflict materials and materials compliance, amongst others. The strength of compliance portals lies in their ability to simplify and automate the process of acquiring data from different suppliers. However, their main weakness is that they require commitment from suppliers who might be reluctant to share information if they are not obliged by law to share it.

\section{Conclusions and Moving Forward}

The work detailed in this paper focused on the initial steps in the development of a PLM based web portal, specifically exploring possible portal architectures based on their strategic alignment with the goals of firms using it. All the work that was carried out was informed by knowledge and information gained from researching past and existing web portals, a survey into supplier collaboration projects and an in-depth case 
study potential G.EN.ESI platform user. Four different architectures, based on the number of companies interacting with a single portal, were generated using scenario planning and presented as a series of potential gains and barriers. Ten use cases which provided a range of possible cases that could arise within the four generated scenarios were created allowing for the viability portal architectures to be tested using the technique of wind-tunnelling. The results from the wind tunnelling ranked Scenario 1 as highly viable, Scenario 3 as medium-high viability and Scenarios 2 and 4 as medium viability. In Scenario 1, multiple buyers and multiple suppliers interact with a single web portal. The consolidation in buyers and suppliers mitigates any bargain-power related issues that might arise, while making way for the possible development of an industry wide information sharing standard. Looking in to existing portals, the presence of compliance portals highlighted that web portals are now being used as more than e-procurement tools.

Moving forward, the project aims to gain a better understanding of supplier collaboration in new product development through the use of the portal by exploring the nature of the information being shared, the roles that users of the portal play (those who initiate it vs. those who participate in it) and any competitive conditions associated with the use of the portal.

\section{References}

Bacallan, J.: Greening the Supply Chain. Business and Environment 6 (2000)

Boks, C.: The Soft Side of Eco-design. Journal of Cleaner Production 14 (2006)

Conner, K., Prahalad, C.K.: A Resource-Based Theory View of the Firm: Knowledge vs Opportunism. Organisational Science 7 (1996)

Ellram, L., Tate, W., Carter, C.: Applying 3DCE to Environmentally Responsible Manufacturing Practices. Journal of Cleaner Energy 16, 1620-1631 (2008)

Fine, C.H.: Clockspeed: Winning Industry Control in the Age of Temporary Advantage. Perseus Books, Massachusetts (1998)

Foresight, Scenario Planning. Foresight Horizon Scanning Center (2009)

Handfield, R., Melnyk, S., Calantone, R., Curkovic, S.: Integrating Environmental Concerns into the Design Process: The Gap between Theory and Practice. IEEE Transactions on Engineering Management 48, 189-208 (2001)

Kanter, R.M.: Collaborative Advantage. Harvard Business Review 72 (1994)

Lofthouse, V.: Eco-design tools for designers: Defining the requirements. Journal of Cleaner Production 14, 1386-1395 (2006)

McIvor, R., McHugh, M.: Collaborative buyer supplier relations: Implications for organization change management. Strategic Change 9, 221-236 (2000)

Roberts, B.: Web Portals pen doors to one-step services. HR Magazine 44 (1999)

Schoemaker, P.: Scenario Planning: A Tool for Strategic Thinking. Sloan Management Review, 25-40 (Winter 1995)

Sudarsan, R., Fenves, S., Sriram, R., Wang, F.: A Product Information Modelling Framework for PLM. Computer-Aided Design 37 (2005)

Yu, Z., Yan, H., Cheng, T.C.: Benefits of Information Sharing with Supply Chain Partnerships. Industrial Management and Data Systems 101 (2001) 